\title{
Construction of pIRES2 vector encoding truncated NS3 of HCV and IL-18 for DNA vaccine studies
}

\author{
Zavvar $\mathbf{M}^{1}$, Moshiri $\mathrm{A}^{2}$, Motevali $\mathrm{F}^{1}$, Pouriayevali $\mathrm{MH}^{3^{*}}$, Bahramali $\mathrm{G}^{\mathbf{1}^{*}}$ \\ ${ }^{1}$ Department of Hepititis and AIDS, Pasteur Institute of Iran, Tehran, Iran. \\ ${ }^{2}$ Department of biotechnology, faculty of advanced technologies in medicine, shahid beheshti university of medical sciences, \\ tehran, iran \\ ${ }^{3}$ Faculty of Medical Science, Tarbiat Modares University (TMU), Tehran, Iran.
}

\begin{abstract}
Introduction: Long term complications of hepatitis $C$ virus (HCV) infection include fibrosis, cirrhosis, and hepatocellular carcinoma and although the disease is treatable with the newly introduced direct acting antivirals, but factors such as drug resistance, viral genotype and adverse effects can limit the effectiveness of therapy. Therefore, development of effective, safe and affordable prophylactic and therapeutic vaccines is a global goal. This study was undertaken for construction of a vector containing the coding region for a truncated form of nonstructural protein 3 (NS3) of HCV and IL-18 cytokine gene fused to murine Fcy2a as molecular adjuvant for assessment as a DNA vaccine candidate. Methods: A truncated form of NS3 was amplified and cloned in pIRES2 containing IL-18 hybrid gene. Expression of the truncated NS3 and bioactivity of IL-18 fusion protein were assessed in transfected HEK293 T cells. Physicochemical properties determination, secondary structure, 3D modeling and T-cell epitope predictions were made using various online tools. Results: In silico analysis of truncated NS3 predicted a molecular weight of 32.35 $\mathrm{kDa}$ containing epitopic regions with the highest scores for binding to MHC complex. 3D model of IL-18 showed that fusion with murine Fc had not impaired the structure. HEK293 T cells were successfully transfected with pIRES-IL18NS3 construct and expression of truncated NS3 with an apparent molecular weight of approximately $32 \mathrm{kDa}$ was confirmed by Western blotting. Conclusion: Despite the advantages, widespread use of DNA vaccination has been hampered by low immunogenicity. Truncated NS3 was expressed albeit in low amount with bioactive IL-18 fusion proteinas a molecular adjuvant Immunogenicity assessment of this novel combination as a DNA vaccine candidate is underway.
\end{abstract}

KEYWORDS: HCV, NS3, IL18, DNA vaccine, Adjuvant.

\section{INTRODUCTION}

Hepatitis $C$ virus $(\mathrm{HCV})$ is a hepato-tropic member of Flaviviridae viral family with an approximately $9.5 \mathrm{~kb}$ positive stranded RNA genome. As has been estimated by the World Health Organization (WHO) 185 million individuals are infected with the virus throughout the world with higher prevalence in the Middle East $[1,2]$. The first 6 months of the disease is virtually symptomless and constitutes the acute phase

*Corresponding Author: Mohammad Hasan Pouriayevali, Ph.D Candidate; Faculty of Medical Science, Tarbiat Modares University (TMU), Tehran, Iran.

Email: mhpouriayevali@yahoo.com

Tel/Fax: (+98) 2166969291

*Co- Corresponding Author: Golnaz Bahramali, Ph.D Candidate;

Department of Hepititis and AIDS, Pasteur Institute of Iran, Tehran, Iran.

Email: gbahramali@gmail.com

Tel/Fax: (+98) 2166969291 during which, the virus is cleared in an estimated $20-30 \%$ of the Infects without any therapy, whereas in the remaining $70-80 \%$ of newly infected, the disease becomes chronic with long-term complications including fibrosis, cirrhosis, and hepatocellular carcinoma [3]. Although HCV infection can be treated with the newly introduced direct acting antivirals, but factors such as drug resistance, viral genotype, host factors, cost, access, efficacy and adverse effects can limit the effectiveness of therapy. Therefore, development of effective, safe and affordable prophylactic and therapeutic vaccines against $\mathrm{HCV}$ infection remains a global goal. Although induction of immunity after spontaneous viral clearance in primary $\mathrm{HCV}$ infection suggests that development of an effective vaccine is possible, but despite all efforts no such vaccine has been developed yet [4]. There is ample clinical evidence showing a major role for both CD4+ and CD8+ T cells in clearing primary and secondary $\mathrm{HCV}$ infections particularly the CD4+ immune 
responses to non-structural protein 3 (NS3) in acute phase clearance [5]. NS3 is a $69 \mathrm{kDa}$ multi-functional hydrophobic protein with serine protease and nucleoside triphosphatase (NTPase)/RNA helicase activities involved in processing of the viral polyprotein and viral RNA replication. It has been shown that NS3 induces immune responses capable of clearing HCV, making it an attractive candidate for a therapeutic vaccine [5-8]. DNA vaccination technology is a promising platform offering a number of advantages over traditional immunization such as safety and cost, but the main advantage of this vaccine modality is the ability of DNA vaccines to stimulate both arms of adaptive immune system, eliciting strong CLT and B-cell immunity [5]. However, despite all the advantages, DNA vaccines have not had widespread usage and the major impediment to their use has been their low immunogenicity in humans [5]. One of the strategies used for overcoming this obstacle has been the co-administration of immunomodulatory cytokines such as IL-18. Transfected cells can express the molecular adjuvant which can modify the immune response towards each particular pathogen [9]. IL-18 is expressed as a 24 $\mathrm{kDa}$ inactive pro-IL-18 requiring conversion to a secreted 18 $\mathrm{kDa}$ active protein by enzymatic digestion with cysteine caspase-1 [10]. Recently it has been shown that priming of natural killer lymphocytes with IL-18 results in memory-like properties and enhanced IFN- $\gamma$ production [11].

Cloning the sequence for mature IL-18 renders the polypeptide non-secretory necessitating introduction of a signal sequence for the expressed protein to be secreted. Therefore in this study a previously constructed vector containing matureIL-18 fused to the signal sequence of immunoglobulin kappa (IgK), and mice IgG1 Fc fragment (murine Fcy2a) was used [12] for cloning of a truncated form of NS3 for assessment as a DNA vaccine candidate.

\section{MATERIALS and METHODS}

PCR primers were synthesized by Pishgam Biotech (Iran). All enzymes and other routine molecular biology reagents were purchased from Thermo Scientific (Germany). RNA extraction, plasmid purification and cDNA synthesis kits were from Qiagen (Valencia, CA).

Bioinformatics analysis of NS3 and IL18 fragments

In this study, protein sequences of NS3, mature IL-18 and murine Fcy2a fragment were derived from national center for biotechnology information (hhtp://www.ncbi.nlm.nih.gov). Physicochemical properties of the proteins were computed using ProtParam online tool (http://web.expasy.org/protparam/) [13].

Self-optimized prediction method with alignment (SOPMA) (http:/npsa-pbil.ibcp.fr/cgibin/npsa_automat.pl?page=/NPSA/npsa_sopma.html) was used for secondary structure prediction of IL18 and mice IgG1 FC fragment (murine Fcy2a) fusion protein and for calculating the number of a-helix, $b$ sheets, turns random coils, and extended strands [14]. The 3D model was obtained using the Threading Assembly Refinement(I-TASSER)online server program which generates 3D models along with their confidence score (C-Score). Calculation of this score which is an evaluation of the quality of the predicted model is based on the significance of threading template alignments and the convergence parameters of the structure assembly simulations [15]. Stereo chemical analysis of obtained 3D protein models were performed using PROCHECK, ERRAT, VERIFY 3D programs for structural analysis which were verified using
SAVES server (http://nihserver.mbi.ucla.edu/SAVES and RAM-PAGE) [16-19].

SMART (Simple Modular Architecture Research Tool, http://smart.embl.de/) online software and InterPro database (protein sequence analysis \& classification, http://www.ebi.ac.uk/interpro/) were used for IL-18 fusion protein domain prediction [20-21].

T-cell epitope regions of NS3 protein were predicted using the web servers SYFPEITHI (http://www.syfpeithi.de/) and ProPred-I (http://www.imtech.res.in/raghava/propred1/) [2223]. The results obtained showed a sorted list of nine amino acid substrings of the submitted antigen sequence for affinity calculations.

\section{Construction of the expression vector}

Plasma from a confirmed, chronically infected HCV-1 patient was used for amplification of NS3 fragment. Total RNA extraction and cDNA synthesis were performed as previously described [12] and the 927bpNS3 fragment was amplified using F-NS3: 5'-CTG CAG ATC CAC AAC CAT GGG TCC TGT CAT CC-3' and R-NS3: 5'-ATA GTT TAG CGG CCG CTC AGA GCT CGT CGC A-3'as forward and reverse primers respectively. Pfu DNA polymerase was used in 35 cycle amplification with initial denaturation at $94{ }^{\circ} \mathrm{C}$ for $5 \mathrm{~min}$ followed by denaturation at $95^{\circ} \mathrm{C}$ for $30 \mathrm{~s}$, annealing at $52^{\circ} \mathrm{C}$ for $30 \mathrm{~s}$, elongation at $72{ }^{\circ} \mathrm{C}$ for $60 \mathrm{~s}$, and the program ended with a final extension at $72{ }^{\circ} \mathrm{C}$ for $10 \mathrm{~min}$.

Amplified product was digested with BstXI and NotI and cloned in similarly digested pIRES2 (Clontech, USA) replacing the sequence for EGF protein in the vector. The construct was transformed into competent E. coli $\mathrm{DH} 5 \alpha$ and transformants were selected on Luria Bertani (LB) agar plates containing 50 $\mu \mathrm{g} / \mathrm{ml}$ kanamycin (Sigma-Aldrich,USA). The authenticity of the cloned gene was verified by sequencing (SEQLAB, Germany). Construction and cloning of IL-18 fusion protein in pIRES2 vector have been described previously [12].

\section{Expression of NS3 protein in eukaryotic cells}

Human HEK293T cells were grown and maintained in DMEM medium containing $2 \mathrm{mM}$ L-glutamine, $100 \mathrm{U} / \mathrm{ml}$ penicillin, $100 \mathrm{mg} / \mathrm{ml}$ streptomycin (Biosera, France), supplemented with $15 \%$ fetal calf serum (Invitrogen, USA), at $37{ }^{\circ} \mathrm{C}$ under humidified air containing $5 \% \mathrm{CO} 2$ with a daily change of culture medium for 4 days. Plates were seeded with 2.4 x 105 cells, grown to $70 \%$ confluency and transfected with $100 \mathrm{ng}$ of pIRES2-EGFP andpIRES-IL18-NS3 using TurboFect in vitro Transfection Reagent according to the manufacturer's protocol (Thermo Scientific, USA). After $60 \mathrm{~h}$, cells were washed with phosphate-buffered saline (PBS, pH 7.5) and transfection efficiency was analyzed by fluorescent microscopy and SDS-PAEG showing expression of EGFP reporter and NS3 respectively.

\section{Western Blot analysis}

Transfected HEK-293 cells expressing NS3 and untransfected cells (negative control) were washed three times with PBS and lysed with buffer containing $0.5 \mathrm{mM}$ EDTA, $1 \mathrm{mM}$ DTT, $2 \mathrm{mM}$ sodium fluoride, $10 \%$ glycerol, $0.2 \%$ Triton $\mathrm{X}-100$ in PBS $\mathrm{pH}$ 7.4and protease inhibitors (Calbiochem France). Proteins electrophoresed on $12 \%$ polyacrylamide-SDS gels and separated protein bands were transferred to PVDF membranes (Pall, USA) using semi-dry blotting system (Bio-Rad, USA). Membranes were blocked with BSA (3\%) at room temperature for $2 \mathrm{~h}$ and incubated with 1:100 dilution ofmouseanti-NS3 monoclonal antibodies (Abcam, USA) for $1 \mathrm{~h}$. Subsequently, washed membranes were incubated with 1:1000 dilution of 
HRP- conjugated rabbit anti-mouse IgG/HPR (Sigma-Aldrich, USA) as secondary antibody and developed using 3,3'diaminobenzidine (DAB, Bio-Rad USA).

Expression and bioactivity of recombinant murine IL-18 Expression and biological activity of recombinant IL-18 fusion protein in the concentrated cell supernatants of HEK-293 T cells was measured using IL-18 ELISA kit (Bender MedSystems, Austria) and Mouse IFN- $\gamma$ ELISA (U-cytech bioscience, Netherlands) according to manufacturer's instructions as previously described [12].

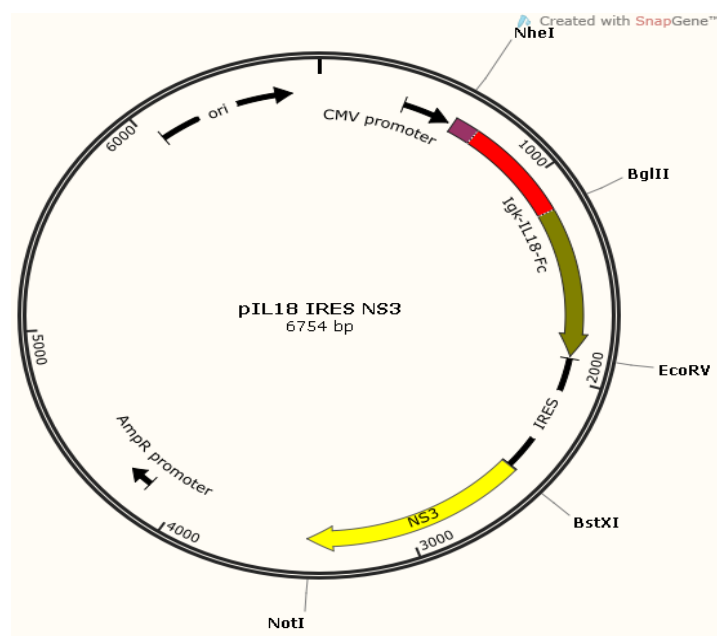

Fig. 1. Schematic presentation of recombinant pIRES-IL18-NS3.

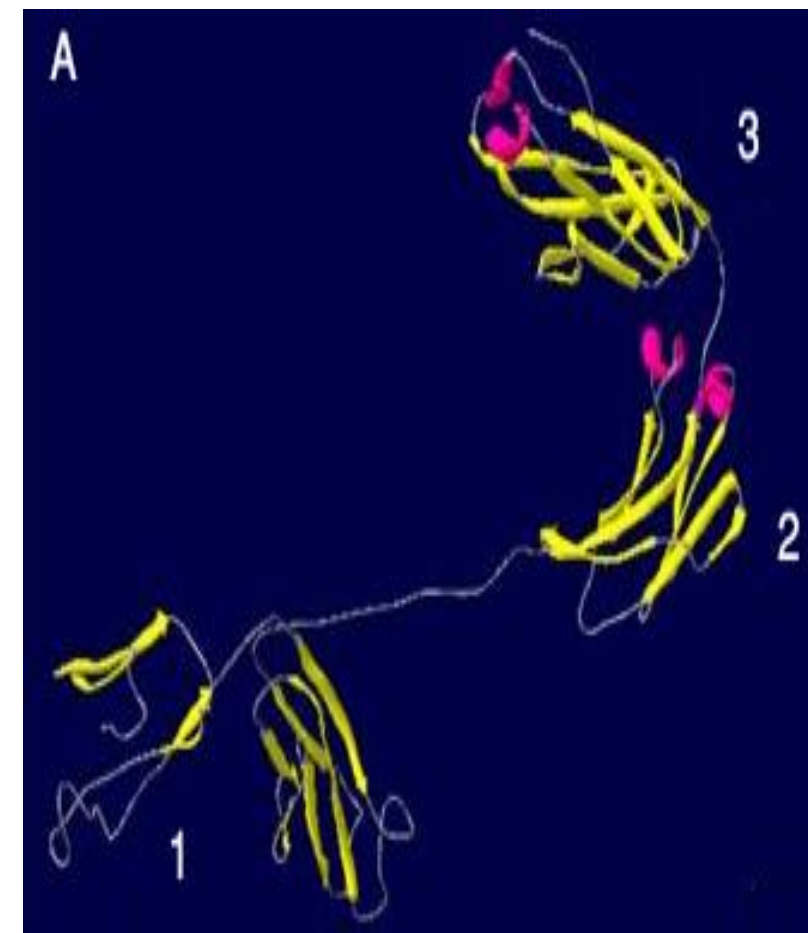

\section{RESULTS}

The primers designed in this study successfully amplified the selected NS3 gene fragment and the authenticity of the product was verified by sequencing. The coding sequence for fulllength NS3 starts from 3623 nucleotide to 4550 of $\mathrm{HCV}$ subtype 1acomplete genome corresponding to amino acids 1027 to 1658 of its polyprotein consensus sequence (NCBI accession number AF009606). The amplified fragment consisted of 120 aa of NS3 N-terminal portion and 189 aa were from domain 1 of NS3 helicase.

In silico evaluation of the 310 amino acids of truncated NS3 fragment showed that there are a large number of hot spot regions in this fragment that can be recognized by MHC class 1. Consensus $\mathrm{T}$ cell epitopes predicted by both SYFPEITHI and ProPred-I methods were combined to achieve a creditable $\mathrm{T}$ cell epitopes prediction for full-length and truncatedNS3. The epitopes from the target region of NS3 protein using SYFPEITHI listed in Table 1 showed the highest score.

Analysis of the physiochemical parameters of this fragment using ProtParam online tool calculated an isoelectric point (pI) of 8.34 and a grand average hydropathicity of 0.051 with an apparent molecular weight of $32.35 \mathrm{kDa}$.

IL-18 fusion protein consisted of 392 amino acids and physiochemical characterization of the protein estimated a molecular weight of $44.5557 \mathrm{kDa}$ and a theoretical isoelectric point of 5.72with the calculated grand average hydropathicity of -0.484 . The total number of positively (Arg+ Lys) and of negatively charged residues (Asp+ Glu) in the polypeptide was 45 and 54 respectively.

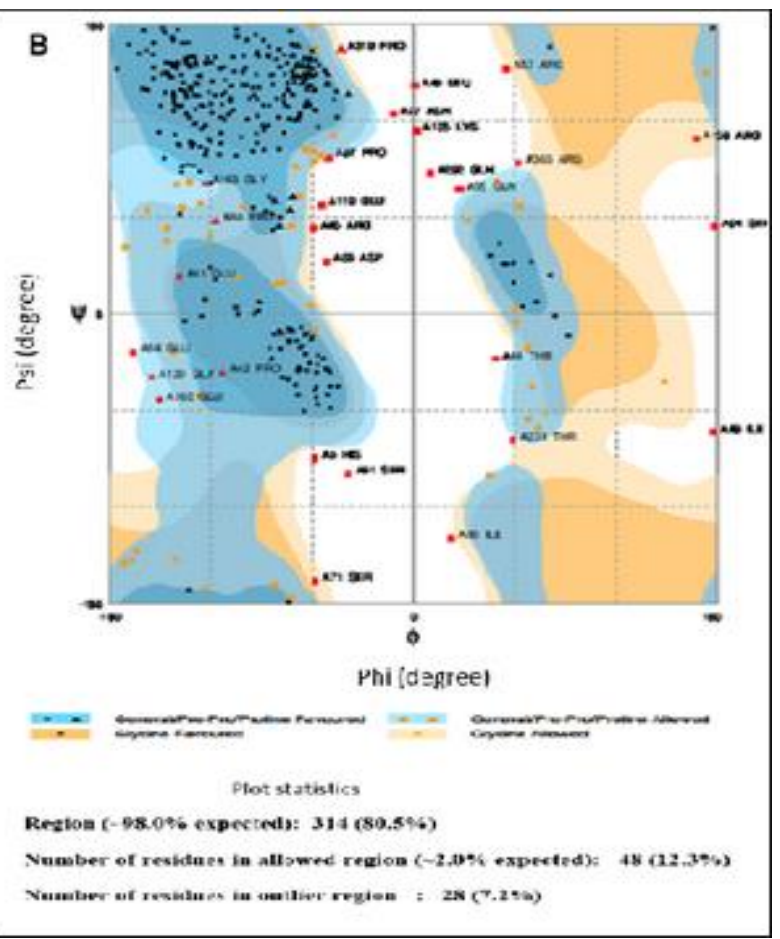

Fig. 2: 3D model of IL-18-murine Fcy2 a fusion protein. A- Predicted 3D model of IL-18-murine Fcy2 a fusion protein obtained from I-TASSER. Image was produced using the Swiss PDB viewer with colors identification as; helices, pink; beta sheets yellow; and all others gray, aa 14-132, IL-1 domain 1, aa 201-276, IG-like domain 2,aa 307-380, IGc1 domain 3 [24]. B- Ramachandran plot of IL18 fusion protein predicted by RAMPAGE, showing residues in favored, allowed outlier regions. 
3D structure of the IL-18 fusion protein was predicted using the I-TASSER online server and the best predicted structure with the maximum confidence score $(\mathrm{C}$-Score -0.44$)$ was selected (Fig. 2A). The predicted 3D model showed that the cytokine had 3 well defined domains and fusion with murine $\mathrm{Fc}$ to the $\mathrm{C}$ terminal had not impaired the structure. Ramachandran plot for the model showed $80.5 \%$ of the protein residues in the favored, $12.3 \%$ residues in allowed and $7.2 \%$ residues in outlier regions (Fig. 2B).

Expression and bioactivity of IL-18

Expression of IL-18-murine Fcy2afusion protein was assayed and the amount of the expressed protein was estimated as 250 $\mathrm{pg} / \mathrm{ml}$ with bioactivity of approximately $27 \mathrm{pg} / \mathrm{ml}$ similar to previously reported data [12]. Detection of IL-18 and its bioactivity in the HEK293T cell culture was taken as evidence of IL-18 secretion.

\section{NS3 expression on eukaryote cells}

Analysis of the transfected cells lysate by SDS-PAGE and subsequent Western blotting confirmed low expression of NS3

protein by HEK293T cells (Fig.3A).Apparent molecular weight of the expressed protein was approximately $32 \mathrm{kDa}$, similar to that calculated by ProtParam online tool.

Table 1. list of predicted T-cell epitopes of NS3 fragment with the highest score using SYFPEITHI.

\begin{tabular}{|c|c|c|}
\hline Position & Sequence & Score \\
\hline 199 & "TYSTYGKFL" & 28 \\
\hline 232 & "SILGIGTVL" & 24 \\
\hline 7 & "MYTNVDQDL" & 23 \\
\hline 173 & "AYMSKANGV" & 22 \\
\hline 150 & "AYAAQGYKV" & 21 \\
\hline 225 & "CHSTDATSI" & 20 \\
\hline 261 & "PFYGKAIPL" & 20 \\
\hline 129 & "SFQVAMLMA" & 19 \\
\hline 190 & "RTITGGSPI" & 18 \\
\hline 228 & "TDATSILGI" & 18 \\
\hline
\end{tabular}
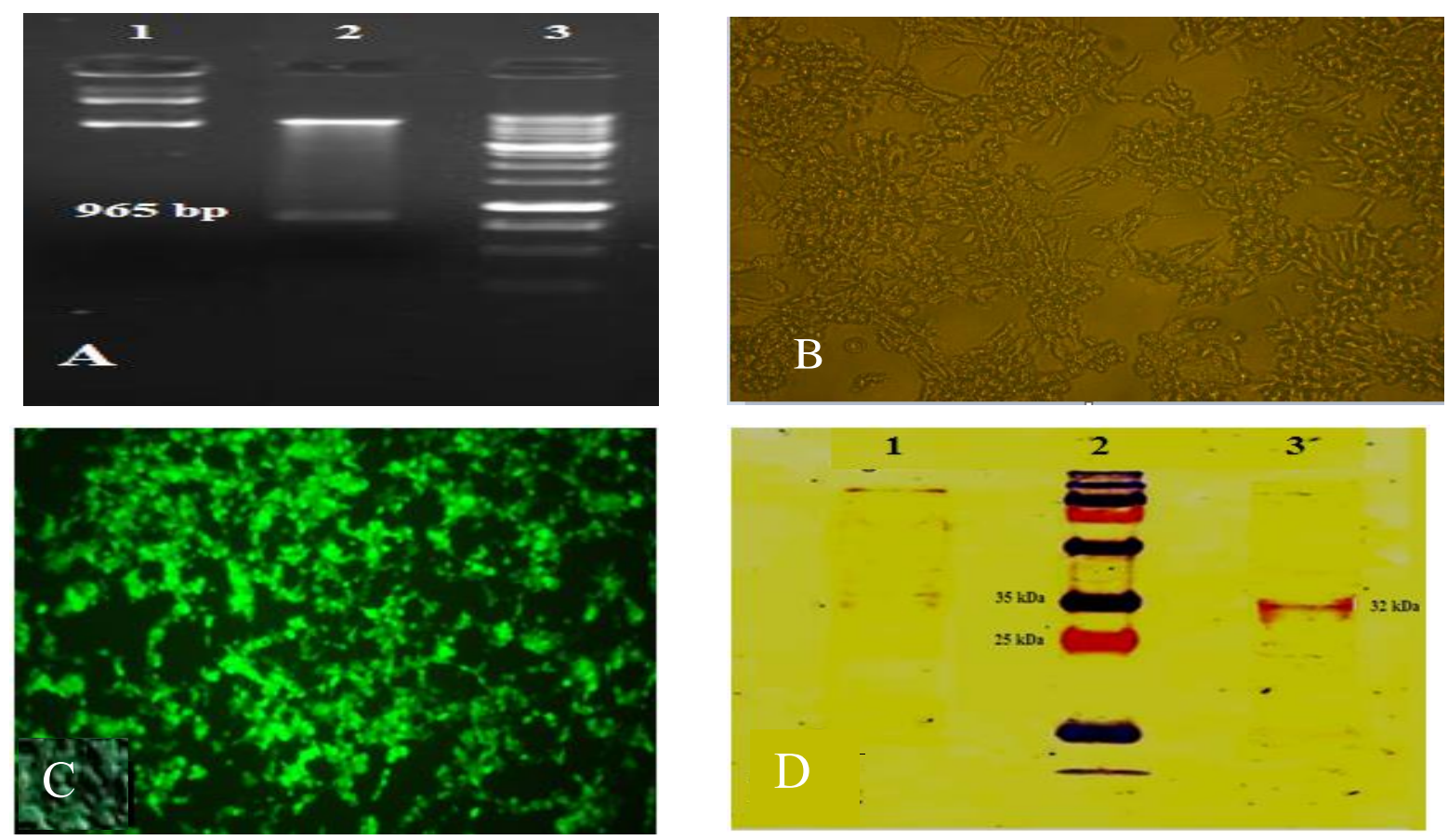

Figure 3: A) Restriction enzyme analysis, Lane 1: undigested pIRES2IL18-NS3. Lane 2: double digested pIRES2IL18-NS3 with BstxI and NotI. Lane 3: 1 kb DNA Ladder. B)Light microscopy image of untransfected HEK293 T cells at 70\% confluency at the time of transfection. C)Fluorescent microscopy image of HEK293 T cells transfected with plasmid containing gfp as reporter gene for assessing transfection efficiency. D) Western blot analysis ofNS3 expression in HEK293T using anti-NS3 monoclonal antibodies. Lane 1: untransfected cells. Lane 2: protein molecular weight marker. Lane 3: lysate of transfected cells expressing truncated NS3.

Expression of the protein was confirmed by Western blotting with mouse anti-NS3 monoclonal antibody (Fig. 3D).

\section{DISCUSSION}

Spontaneous clearance of $\mathrm{HCV}$ in a small portion of the infected hosts has spurred the search for prophylactic and therapeutic vaccines against this viral infection. NS3 protein is one of the more genetically conserved antigens in the highly variable $\mathrm{HCV}$ genome and the importance of its specific $\mathrm{T}$ cell responses in clearance of acute infection has been shown[25].The protease and helicase activities of the protein have been mapped to the $\mathrm{N}$-terminal one third and $\mathrm{C}$-terminal two thirds of the full-length polypeptide respectively[26].The enzymatic activities of NS3 while pivotal in viral lifecycle may interfere with host defense mechanism, therefore in this study a truncated form of the protein lacking protease and helicase activity was used [7]. Truncated fragment however, contained a number of $\mathrm{T}$ cell epitopes that ranked very high in in silico analysis (Table 1). Comparison of epitopic regions in fulllength NS3 with the truncated form showed that epitopic domains with the highest score in full-length protein were located in the truncated NS3.Expression level of the $\triangle \mathrm{NS} 3$ was not very high despite the reduction of cysteine residues from 20 in to 9 (Fig. 3) which was similar to the result reported by Hosseini et al. [27]. 
Calculated grand average hydropathicity of the selected segment was slightly higher compared to the full form which could have adversely affected the expression level of the heterologous protein. Recent studies have shown that the presence of the NS4A cofactor improves expression and immunogenicity of the expressed protein and the higher immunogenicity was attributed to the higher expression of NS3[28].Therefore increasing the expression level of the truncated NS3 requires production-enhancing strategies which are underway to improve the suitability of the proposed candidate antigen. However, preliminary data obtained have indicated that the combination of truncated NS3 and IL-18 used in this study is capable of stimulating an adequate immunological response and the immune response is protective (data not shown).

The multifunctional IL-18 cytokine strongly stimulates innate and adaptive immune systems, boosting Ag-specific $\mathrm{T}$ cell responses and can be used as an effective adjuvant in DNA vaccination [9].Fusion of murine Fcy2a fragment added nearly $26 \mathrm{kDa}$ to molecular weight of IL-18 and reduced the grand average hydropathicity of the protein slightly. Several advantages have been cited for fusion of murine Fcy2a to cytokines including increased avidity, prolonged circulating half-life due to molecular size increase and reduced renal clearance. Furthermore, fusion with Fc results in alteration of complement-mediated and antibody dependent cell-mediated cytotoxicity, enhancing the effects of IL-18 as a molecular adjuvant [29]. The strong stimulatory effect exerted by IL-18 fusion protein might compensate for the low expression level observed for the candidate antigen.

In conclusion the combined use of truncated NS3 and IL-18 has the potential to be used as candidate therapeutic DNA vaccine.

\section{ACKNOWLEDGEMENT}

We thank A. Memarnejadian, S.M.Sadat and R.Vahabpour for their kind help and generous advice. This research was supported by grant no.478 from Pasteur institute of Iran.

\section{CONFLICT OF INTEREST}

The authors declare that they have no conflict of interest.

\section{REFERENCES}

1. Mohd Hanafiah K, Groeger J, Flaxman AD, Wiersma ST. Global epidemiology of hepatitis $\mathrm{C}$ virus infection: new estimates of age-specific antibody to HCV seroprevalence. Hepatology. 2013;57(4):1333-42. doi:10.1002/hep.26141.

2. Thomas DL. Global control of hepatitis C: where challenge meets opportunity. Nat Med. 2013;19(7):850-8. doi:10.1038/nm.3184.

3. Abdel-Hakeem MS, Shoukry NH. Protective immunity against hepatitis C: many shades of gray. Front Immunol. 2014;5:274. doi:10.3389/fimmu.2014.00274.

4. Feinstone SM, Hu DJ, Major ME. Prospects for prophylactic and therapeutic vaccines against hepatitis C virus. Clin Infect Dis. 2012;55 Suppl 1:S25-32. doi:10.1093/cid/cis362.

5. Flingai S, Czerwonko M, Goodman J, Kudchodkar SB, Muthumani K, Weiner DB. Synthetic DNA vaccines: improved vaccine potency by electroporation and co-delivered genetic adjuvants. Front Immunol. 2013;4:354. doi:10.3389/fimmu.2013.00354.

6 . Beran RK, Serebrov V, Pyle AM. The serine protease domain of hepatitis $\mathrm{C}$ viral NS3 activates RNA helicase activity by promoting the binding of RNA substrate. J Biol Chem. 2007;282(48):34913-20. doi:10.1074/jbc.M707165200.

7. Ratnoglik SL, Jiang DP, Aoki C, Sudarmono P, Shoji I, Deng L et al.
Induction of cell-mediated immune responses in mice by DNA vaccines that express hepatitis C virus NS3 mutants lacking serine protease and NTPase/RNA helicase activities. PLoS One. 2014;9(6):e98877. doi:10.1371/journal.pone.0098877.

8. Lang Kuhs KA, Ginsberg AA, Yan J, Wiseman RW, Khan AS, Sardesai NY et al. Hepatitis C virus NS3/NS4A DNA vaccine induces multiepitope $\mathrm{T}$ cell responses in rhesus macaques mimicking human immune responses [corrected]. Mol Ther. 2012;20(3):669-78. doi:10.1038/mt.2011.188.

9. Wan YY, Flavell RA. How diverse--CD4 effector T cells and their functions. J Mol Cell Biol. 2009;1(1):20-36. doi:10.1093/jmcb/mjp001.

10. Fantuzzi G, Dinarello CA. Interleukin-18 and interleukin-1 beta: two cytokine substrates for ICE (caspase-1). J Clin Immunol. 1999;19(1):1-11.

11. Romee R, Schneider SE, Leong JW, Chase JM, Keppel CR, Sullivan RP et al. Cytokine activation induces human memory-like NK cells. Blood. 2012;120(24):4751-60. doi:10.1182/blood-2012-04-419283.

12. Pouriayevali MH, Memarnejadian AR, Sadat SM, Zavvar M, Siadat SD, Hartoonian C, Aghasadeghi MR. Designing and construction of bicistronic plasmid pIRES-Igk/mIL18/Fc: potential implications for vaccine investigations. Modarres J Med Sci 2011; 14; 13-23

13. Wilkins MR, Gasteiger E, Bairoch A, Sanchez JC, Williams KL, Appel $\mathrm{RD}$ et al. Protein identification and analysis tools in the ExPASy server. Methods Mol Biol. 1999;112:531-52.

14. Geourjon C, Deleage G. SOPMA: significant improvements in protein secondary structure prediction by consensus prediction from multiple alignments. Comput Appl Biosci. 1995;11(6):681-4.

15. Zhang Y. I-TASSER server for protein 3D structure prediction. BMC Bioinformatics. 2008;9:40. doi:10.1186/1471-2105-9-40.

16. Laskowski RA, MacArthur MW, Moss DS, Thornton JM. PROCHECK -a program to check the stereochemical quality of protein structures. J App Cryst 1993; 26; 283-291.

17. Colovos C, Yeates TO. Verification of protein structures: patterns of nonbonded atomic interactions. Protein Sci. 1993;2(9):1511-9. doi:10.1002/pro.5560020916.

18. Bowie JU, Luthy R, Eisenberg D. A method to identify protein sequences that fold into a known three-dimensional structure. Science. 1991;253(5016):164-70.

19. Lovell SC, Davis IW, Arendall WB, de Bakker PIW, Word JM, Prisant MG, Richardson JC and Richardson DC. Structure validation by C-alpha geometry, phi,psi and $\mathrm{C}$ beta deviation. Proteins: Structure, Function and Genetics. 2002; 50; 437-450.

20. Letunic I, Doerks T, Bork P. SMART: recent updates, new developments and status in 2015. Nucleic Acids Res. 2015;43(Database issue):D257-60. doi:10.1093/nar/gku949.

21. Mitchell A, Chang HY, Daugherty L, Fraser M, Hunter S, Lopez R et al. The InterPro protein families database: the classification resource after 15 years. Nucleic Acids Res. 2015;43(Database issue):D213-21. doi:10.1093/nar/gku1243.

22. Rammensee H, Bachmann J, Emmerich NP, Bachor OA, Stevanovic S. SYFPEITHI: database for MHC ligands and peptide motifs. Immunogenetics. 1999;50(3-4):213-9.

23. Singh H, Raghava GP. ProPred1: prediction of promiscuous MHC Class-I binding sites. Bioinformatics. 2003;19(8):1009-14.

24. Guex N, Peitsch MC. SWISS-MODEL and the Swiss-PdbViewer: an environment for comparative protein modeling. Electrophoresis. 1997;18(15):2714-23. doi:10.1002/elps.1150181505.

25. Aberle JH, Formann E, Steindl-Munda P, Weseslindtner L, Gurguta C, Perstinger G et al. Prospective study of viral clearance and CD4(+) T-cell response in acute hepatitis $\mathrm{C}$ primary infection and reinfection. J Clin Virol. 2006;36(1):24-31. doi:10.1016/j.jcv.2005.12.010.

26. Raney KD, Sharma SD, Moustafa IM, Cameron CE. Hepatitis C virus non-structural protein 3 (HCV NS3): a multifunctional antiviral target. J Biol Chem. 2010;285(30):22725-31. doi:10.1074/jbc.R110.125294.

27. Hosseini SY, Sabahi F, Moazzeni SM, Modarressi MH, Saberi Firoozi M, Ravanshad M. Construction and preparation of three recombinant adenoviruses expressing truncated NS3 and core genes of hepatitis C virus for vaccine purposes. Hepat Mon. 2012;12(8):e6130. doi:10.5812/hepatmon.6130.

28. Frelin L, Alheim M, Chen A, Soderholm J, Rozell B, Barnfield C et al. Low dose and gene gun immunization with a hepatitis $\mathrm{C}$ virus nonstructural (NS) 3 DNA-based vaccine containing NS4A inhibit NS3/4A-expressing tumors in vivo. Gene Ther. 2003;10(8):686-99. doi:10.1038/sj.gt.3301933. 29. Vazquez-Lombardi R, Roome B and Christ D. Molecular engineering of therapeutic cytokines. Antibodies 2013; 2; 426451.doi:10.3390/antib2030426. 